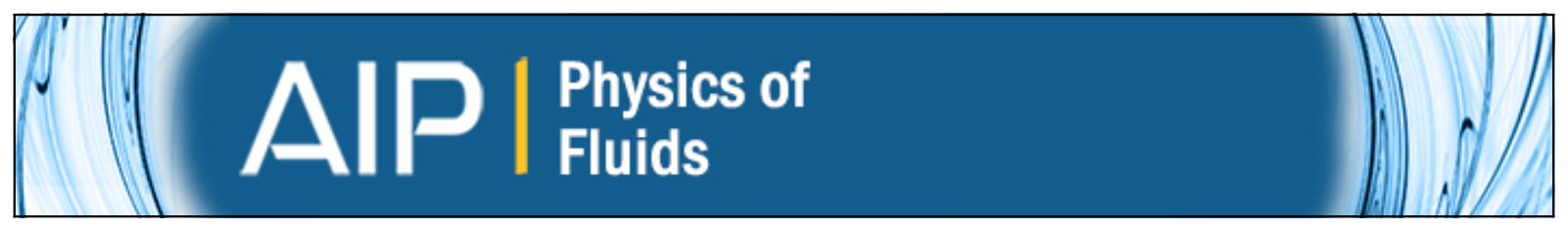

\title{
Instability of vortex pair leapfrogging
}

Laust Tophøj and Hassan Aref

Citation: Physics of Fluids (1994-present) 25, 014107 (2013); doi: 10.1063/1.4774333

View online: http://dx.doi.org/10.1063/1.4774333

View Table of Contents: http://scitation.aip.org/content/aip/journal/pof2/25/1 ?ver=pdfcov

Published by the AIP Publishing

Copyright by the American
Institute of Physics.
Instability of vortex pair
leapfrogging
Tophøj, Laust and Aref, Hassan,
Physics of Fluids (1994-
present), 25, 014107 (2013),
DoI:http://
dx.doi.org/10.1063/1.4774333

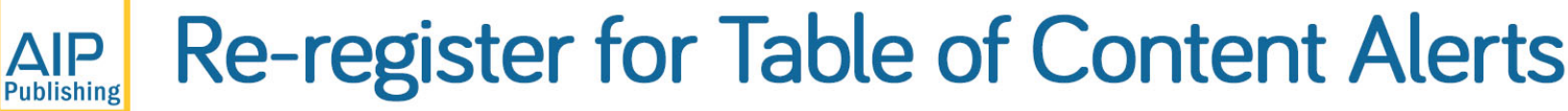




\title{
Instability of vortex pair leapfrogging
}

\author{
Laust Tophøj ${ }^{1,2, a)}$ and Hassan Aref ${ }^{2,3}$ \\ ${ }^{1}$ Department of Physics, Technical University of Denmark, Kongens Lyngby, \\ DK-2800, Denmark \\ ${ }^{2}$ Center for Fluid Dynamics, Technical University of Denmark, Kongens Lyngby, \\ DK-2800, Denmark \\ ${ }^{3}$ Department of Engineering Science and Mechanics, Virginia Tech, Blacksburg, \\ Virginia 24061, USA
}

(Received 1 March 2012; accepted 28 September 2012; published online 30 January 2013)

Leapfrogging is a periodic solution of the four-vortex problem with two positive and two negative point vortices all of the same absolute circulation arranged as co-axial vortex pairs. The set of co-axial motions can be parameterized by the ratio $0<\alpha<1$ of vortex pair sizes at the time when one pair passes through the other. Leapfrogging occurs for $\alpha>\sigma^{2}$, where $\sigma=\sqrt{2}-1$ is the silver ratio. The motion is known in full analytical detail since the 1877 thesis of Gröbli and a well known 1894 paper by Love. Acheson ["Instability of vortex leapfrogging," Eur. J. Phys. 21, 269-273 (2000)] determined by numerical experiments that leapfrogging is linearly unstable for $\sigma^{2}<\alpha<0.382$, but apparently stable for larger $\alpha$. Here we derive a linear system of equations governing small perturbations of the leapfrogging motion. We show that symmetry-breaking perturbations are essentially governed by a $2 \mathrm{D}$ linear system with time-periodic coefficients and perform a Floquet analysis. We find transition from linearly unstable to stable leapfrogging at $\alpha=\phi^{2} \approx 0.381966$, where $\phi=\frac{1}{2}(\sqrt{5}-1)$ is the golden ratio. Acheson also suggested that there was a sharp transition between a "disintegration" instability mode, where two pairs fly off to infinity, and a "walkabout" mode, where the vortices depart from leapfrogging but still remain within a finite distance of one another. We show numerically that this transition is more gradual, a result that we relate to earlier investigations of chaotic scattering of vortex pairs [L. Tophøj and H. Aref, "Chaotic scattering of two identical point vortex pairs revisited," Phys. Fluids 20, 093605 (2008)]. Both leapfrogging and "walkabout" motions can appear as intermediate states in chaotic scattering at the same values of linear impulse and energy. (C) 2013 American Institute of Physics. [http://dx.doi.org/10.1063/1.4774333]

\section{INTRODUCTION}

The possibility that two vortex rings with a common axis can "leapfrog" was already mentioned by Helmholtz in his original paper on vortex dynamics. ${ }^{1}$ He wrote (in Tait's translation): "We can now see generally how two ring-formed vortex-filaments having the same axis would mutually affect each other... the foremost widens and travels more slowly, the pursuer shrinks and travels faster, till finally, if their velocities are not too different, it overtakes the first and penetrates it. Then the same game goes on in the opposite order, so that the rings pass through each other alternately." Pipe smokers skilled in blowing smoke rings will often blow two rings in succession demonstrating the

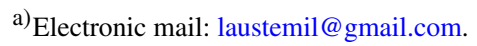


phenomenon. A more precise and often-cited flow visualization experiment was performed many years ago. ${ }^{2}$ A mathematical discussion of the dynamics of thin-filament vortex rings with symmetry can be found in Ref. 3 .

Here we are interested in the two-dimensional counterpart, where two co-axial vortex pairs leapfrog one another, in the special case when the four vortices all have the same absolute circulation. The analytical solution of this periodic motion, when the vortices are represented as point vortices, was derived by Gröbli, ${ }^{4}$ and subsequently by Love, ${ }^{5}$ more than a century ago. Gröbli and Love both found that leapfrogging was possible only if the size ratio of the two pairs at the moment one slips through the other is not too large. To quote from Love's paper: " "... the motion is periodic, if, at the instant when one pair passes through the other, the ratio of the breadths of the pairs is less than $3+2 \sqrt{2}$. When the ratio has this precise value the smaller pair shoots ahead of the larger and widens, while the larger contracts, so that each is ultimately of the same breadth ..., and the distance between them is ultimately infinite. When the ratio in question is greater than $3+2 \sqrt{2}$, the smaller shoots ahead and widens, and the latter falls behind and contracts... When the ratio is less than $3+2 \sqrt{2}$, the motion of the two pairs is similar to the motion described by Helmholtz for two rings on the same axis, and it is probable that there is for this case also a critical condition in which the rings, after one has passed through the other, ultimately separate to an infinite distance, and attain equal diameters." We note that $3+2 \sqrt{2} \approx 5.82843$ and that $(3+2 \sqrt{2})^{-1}=3-2 \sqrt{2} \approx 0.171573$. Here $3-2 \sqrt{2}=\sigma^{2}$, where $\sigma=\sqrt{2}-1$ is called the silver ratio.

In 2000 Acheson published a short paper ${ }^{6}$ in which he reported on numerical simulations where the classical leapfrogging solution had been slightly perturbed, in essence by rotating one of the pairs so that it no longer was symmetric with respect to the centerline of the other. Acheson then found that the leapfrogging motion is unstable and that it breaks down through one of two different modes of instability. He worked in terms of the size ratio $\alpha$ of the smaller pair to the larger at the moment of slip-through. The classical solutions ${ }^{4,5}$ show that leapfrogging requires $\alpha>\sigma^{2}$ $\approx 0.172$. For $\alpha=0.220$, Acheson reproduced the classical solution numerically. Perturbing one of the vortex positions by one part in $10^{6}$, Acheson observed the leapfrogging to cease after a few periods and the four-vortex system disintegrated into two pairs that propagated off to infinity. (See Fig. 1(a) for a similar calculation at $\alpha=0.25$.) For $\alpha=0.310$, and a larger perturbation, a different mode of instability was observed, that Acheson termed the "walkabout" instability. Here one vortex leaves its partner, crosses the centerline, and joins with the other pair in a complicated bounded motion while the entire system continues to move forward. This would then resolve itself back into a leapfrogging motion. Later another "walkabout" event might take place, and so on. (See Fig. 1(b) for a similar calculation at $\alpha=0.305$.) Acheson described his observations in these words: "What appears to happen is that two like-signed vortices occasionally get so close that they revolve rapidly
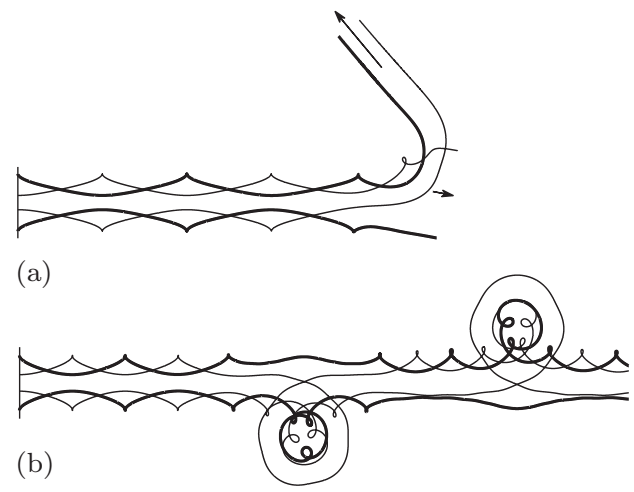

FIG. 1. Instability calculations of leapfrogging similar to those reported by Acheson. ${ }^{6}$ Two different perturbations are shown. Vertical lines indicate the initial positions. (a) A "disintegration" instability for $\alpha=0.25$ perturbed by $\xi_{-}$ $=\eta_{+}=-10^{-6}$. (b) A "walkabout" instability for $\alpha=0.30$ perturbed by $\xi_{-}=0, \eta_{+}=10^{-5}$ (see text for precise definitions). 
around one another, and the combination of the two is then strong enough to sweep one of the other, oppositely-signed, vortices out of its usual path. The three then go off together on a roughly circular excursion, before meeting up again with the fourth, which has been continuing on a more or less straight path in the meantime. 'Walkabout' episodes of this kind take place on seemingly unpredictable sides of the original symmetry axis and at seemingly irregular intervals." In fact, these irregular "walkabout" motions and the sensitive dependence of the directions in which the two pairs propagate in the pair-disintegration mode are both manifestations of this four-vortex problem being chaotic. Acheson ${ }^{6}$ found numerically that there was a transition at the pair-size ratio $\alpha \approx 0.29$, with disintegration instability occurring for smaller $\alpha$ and "walkabout" instability occurring for $\alpha$ closer to 1 . He also found that the "walkabout" instability had a finite range in $\alpha$ and ceased for $\alpha>0.382$. In physical terms ${ }^{6}$ this is "because vortices of like sign are close enough to 'stick together' and avoid disruption by a vortex of opposite sign."

In 2008 the present authors explored scattering experiments in which two identical vortex pairs were sent towards one another and the resulting motion was traced. ${ }^{7}$ We found, among other things, that the intermediate bound states of all four vortices, before they eventually disintegrated into two independent pairs, could contain segments of leapfrogging and also segments that look like Acheson's "walkabout" instability. See Fig. 7 in Ref. 7 and also Fig. 6 later in the present paper. Unfortunately, we were unaware of Acheson's paper ${ }^{6}$ at the time.

Acheson's results ${ }^{6}$ and our results ${ }^{7}$ strongly suggest that the classical leapfrogging solution is an unstable periodic motion of the four-vortex system over part of its parameter range. This statement is amenable to more detailed analysis. Since we are considering a periodic solution, we are led to Floquet theory. ${ }^{8,9}$ The paper is thus laid out as follows: In Sec. II we state the governing equations of motion and establish our notation. The four-vortex system is Hamiltonian. We introduce a new set of canonical variables for it, initially due to Eckhardt and Aref. ${ }^{10}$ These variables reduce the four-degree-of-freedom problem to a two-degree-of-freedom problem and an auxiliary problem that can subsequently be solved. This canonical reduction is particularly important because it allows us to construct perturbations that conserve the total linear impulse of the system.

In Sec. III we summarize those details of the analytical solution for leapfrogging that we need in the following stability analysis. In Sec. IV we write out the linear stability analysis of the canonically reduced system. Although one could have expected this to lead to four coupled ordinary differential equations (ODEs), it turns out that these decouple into two independent systems of two ODEs. These two systems are very similar. In Sec. V we give a brief exposition of Floquet analysis with an eye to its application to our problem in Sec. VI. We evaluate the Floquet exponents numerically. We find that there is indeed a transition from linear instability to stability at a value $\alpha=\alpha_{2} \approx 0.38197$. Deeper analysis reveals that $\alpha_{2}=\phi^{2}$, where $\phi=\frac{1}{2}(\sqrt{5}-1)$ is the golden ratio.

On the other hand, linear analysis does not shed light on Acheson's empirically found value of $\alpha_{1} \approx 0.29$ where there seems to be a change-over from "disintegration" to "walkabout" instability. By precision numerical computations we show that the transition is gradual rather than sharp, in the sense that the instability modes are not confined to either side of the transition. Indeed, we give an example where one leapfrogging motion can suffer either "walkabout" or "disintegration" instability when perturbed in different directions. We conclude that the transition around $\alpha=0.29$ is not a simple, sharp transition in the same sense that the (linear) instability-to-stability transition at $\alpha_{2}$ is. In Sec. VII we consider the limit $\alpha \rightarrow 1$ in detail. In this limit we may analytically evaluate everything in the Floquet stability analysis.

Finally, Sec. VIII contains our conclusions.

\section{EQUATIONS OF MOTION}

We represent the vortices as points in the complex plane, with $z_{1}^{+}$and $z_{2}^{+}$the two positive vortices of circulation $\Gamma, z_{1}^{-}$and $z_{2}^{-}$the two negative of circulation $-\Gamma$. Overbars denote complex 
conjugation. The equations of motion of our point vortex system are

$$
\begin{aligned}
& \frac{\overline{\mathrm{d} z_{1}^{+}}}{\mathrm{d} t}=\frac{\Gamma}{2 \pi \mathrm{i}}\left(\frac{1}{z_{1}^{+}-z_{2}^{+}}-\frac{1}{z_{1}^{+}-z_{1}^{-}}-\frac{1}{z_{1}^{+}-z_{2}^{-}}\right), \\
& \frac{\overline{\mathrm{d} z_{2}^{+}}}{\mathrm{d} t}=\frac{\Gamma}{2 \pi \mathrm{i}}\left(\frac{1}{z_{2}^{+}-z_{1}^{+}}-\frac{1}{z_{2}^{+}-z_{1}^{-}}-\frac{1}{z_{2}^{+}-z_{2}^{-}}\right), \\
& \overline{\frac{\mathrm{d} z_{1}^{-}}{\mathrm{d} t}}=\frac{\Gamma}{2 \pi \mathrm{i}}\left(\frac{1}{z_{1}^{-}-z_{1}^{+}}+\frac{1}{z_{1}^{-}-z_{2}^{+}}-\frac{1}{z_{1}^{-}-z_{2}^{-}}\right), \\
& \frac{\mathrm{d} z_{2}^{-}}{\mathrm{d} t}=\frac{\Gamma}{2 \pi i}\left(\frac{1}{z_{2}^{-}-z_{1}^{+}}+\frac{1}{z_{2}^{-}-z_{2}^{+}}-\frac{1}{z_{2}^{-}-z_{1}^{-}}\right),
\end{aligned}
$$

As with all point vortex problems this system is Hamiltonian.

We change to another set of variables by a canonical transformation due to Eckhardt and Aref: ${ }^{10}$

$$
\begin{aligned}
& \zeta_{0}=\frac{1}{2} \Gamma\left(z_{1}^{+}+z_{2}^{+}-z_{1}^{-}-z_{2}^{-}\right), \\
& \hat{\zeta}_{0}=\frac{1}{2}\left(z_{1}^{+}+z_{2}^{+}+z_{1}^{-}+z_{2}^{-}\right), \\
& \zeta_{+}=\frac{1}{2}\left(z_{1}^{+}-z_{2}^{+}+z_{1}^{-}-z_{2}^{-}\right), \\
& \zeta_{-}=\frac{1}{2} \Gamma\left(z_{1}^{+}-z_{2}^{+}-z_{1}^{-}+z_{2}^{-}\right) .
\end{aligned}
$$

It is also useful to have the inverse transformation,

$$
\begin{aligned}
& z_{1}^{+}=\frac{1}{2}\left[\hat{\zeta}_{0}+\zeta_{+}+\left(\zeta_{0}+\zeta_{-}\right) / \Gamma\right], \\
& z_{1}^{-}=\frac{1}{2}\left[\hat{\zeta}_{0}+\zeta_{+}-\left(\zeta_{0}+\zeta_{-}\right) / \Gamma\right], \\
& z_{2}^{+}=\frac{1}{2}\left[\hat{\zeta}_{0}-\zeta_{+}+\left(\zeta_{0}-\zeta_{-}\right) / \Gamma\right], \\
& z_{2}^{-}=\frac{1}{2}\left[\hat{\zeta}_{0}-\zeta_{+}-\left(\zeta_{0}-\zeta_{-}\right) / \Gamma\right]
\end{aligned}
$$

If we think of the configuration as consisting of two vortex pairs, $\left(z_{1}^{+}, z_{1}^{-}\right)$and $\left(z_{2}^{+}, z_{2}^{-}\right)$, the intra-pair separations are $d_{1}=z_{1}^{+}-z_{1}^{-}$and $d_{2}=z_{2}^{+}-z_{2}^{-}$. The vector connecting vortex pair centers, from pair $\left(z_{2}^{+}, z_{2}^{-}\right)$to pair $\left(z_{1}^{+}, z_{1}^{-}\right)$, is $\zeta_{+}$. The difference between the intra-pair separations, multiplied by $\Gamma$, is $\zeta_{-}=\frac{1}{2} \Gamma\left(d_{1}-d_{2}\right)$. The quantity $\zeta_{0}=\frac{1}{2} \Gamma\left(d_{1}+d_{2}\right)$ is half the linear impulse of the system and is thus a constant of the motion. A sketch of the coordinates is shown in Fig. 2.

The variables $\zeta_{0}$ and $\hat{\zeta}_{0}$ are conjugate in the sense that $\operatorname{Re} \zeta_{0}$ and $\operatorname{Im} \hat{\zeta}_{0}$ are canonically conjugate, as are $\operatorname{Re} \hat{\zeta}_{0}$ and $\operatorname{Im} \zeta_{0}$. Similarly, $\zeta_{ \pm}$are conjugate variables. In particular, since $\zeta_{0}$ is an integral of the motion, $\hat{\zeta}_{0}$ is a cyclic variable that does not appear in the Hamiltonian $H$. Geometrically $\hat{\zeta}_{0}$ is twice the centroid of the vortex positions. This point may be shifted by a translation of the coordinates which, since the vortex circulations sum to 0 , does not change the value of the linear impulse, $\zeta_{0}$, a property that we shall use in what follows. Since only relative positions of the vortices enter the equations of motion, it is intuitively clear that it should be possible to find a subset of equations that do not contain $\hat{\zeta}_{0}$. The canonical transformation in Eqs. (2a) and (2b) achieves this formally.
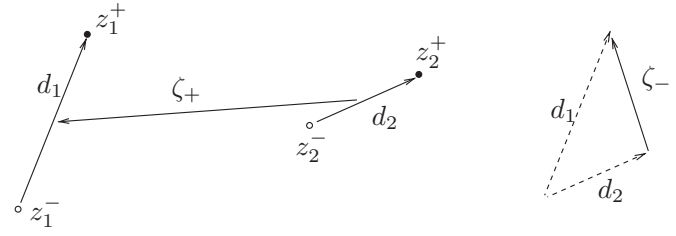

FIG. 2. Positive vortices are at $z_{1}^{+}, z_{2}^{+}$in the complex plane, negative vortices at $z_{1}^{-}, z_{2}^{-}$. Also shown are $\zeta_{ \pm}$, Eqs. (2a). 
Note that a configuration that has the real axis as a symmetry axis, such as the leapfrogging motions, i.e., for which $z_{1}^{-}=\overline{z_{1}^{+}}, z_{2}^{-}=\overline{z_{2}^{+}}$, must have $\zeta_{+}$real and $\zeta_{-}$imaginary. Also note that if we had paired up the vortices as $\left(z_{2}^{+}, z_{1}^{-}\right)$and $\left(z_{1}^{+}, z_{2}^{-}\right)$, we would, in essence, have interchanged the definitions of $\zeta_{ \pm}$(except for a factor of $\Gamma$ ). Hence, the equations of motion written in terms of $\zeta_{ \pm}$ must display this symmetry.

The canonical transformation implies that the equations for $\zeta_{+}$and $\zeta_{-}$form a closed twodegree-of-freedom dynamical system, in which the constant $\zeta_{0}$ enters as a parameter. According to Eq. (3.14) of Ref. 10, the Hamiltonian of this reduced system is

$$
H=-\frac{\Gamma^{2}}{2 \pi} \log \left|\frac{1}{\zeta_{0}^{2}-\zeta_{+}^{2}}-\frac{1}{\zeta_{0}^{2}-\zeta_{-}^{2}}\right|
$$

The resulting equations of motion are

$$
\begin{aligned}
& \frac{\overline{\mathrm{d} \zeta_{+}}}{\mathrm{d} t}=\frac{\Gamma^{2}}{\mathrm{i} \pi} \zeta_{-}\left(\frac{1}{\zeta_{-}^{2}-\zeta_{+}^{2}}+\frac{1}{\zeta_{0}^{2}-\zeta_{-}^{2}}\right), \\
& \frac{\overline{\mathrm{d} \zeta_{-}}}{\mathrm{d} t}=\frac{\Gamma^{2}}{\mathrm{i} \pi} \zeta_{+}\left(\frac{1}{\zeta_{+}^{2}-\zeta_{-}^{2}}+\frac{1}{\zeta_{0}^{2}-\zeta_{+}^{2}}\right)
\end{aligned}
$$

and also,

$$
\frac{\overline{\mathrm{d} \hat{\zeta}_{0}}}{\mathrm{~d} t}=\frac{\Gamma^{2}}{\mathrm{i} \pi} \zeta_{0}\left(\frac{1}{\zeta_{+}^{2}-\zeta_{0}^{2}}+\frac{1}{\zeta_{-}^{2}-\zeta_{0}^{2}}\right)
$$

The case $\zeta_{0}=0$ is integrable ${ }^{10}$ but not of particular interest for the present considerations. Assuming $\zeta_{0} \neq 0$, we scale $\zeta_{ \pm}$and $\hat{\zeta}_{0}$ by $-i \zeta_{0}$, but again call the scaled variables $\zeta_{ \pm}$and $\hat{\zeta}_{0}$. This scaling guarantees that if the vortices initially are placed on the $y$-axis, so that the leapfrogging motion would propagate along the $x$-axis, then $\zeta_{ \pm}$and $\hat{\zeta}_{0}$ are proportional to their scaled counterparts with a real coefficient of proportionality. We now obtain a common pre-factor $\Gamma^{2} / \pi\left|\zeta_{0}\right|^{2}$ on the right-hand sides of the preceding equations of motion. We may choose units of length and time such that this common pre-factor is 1 . The re-scaled equations of motion are then simply

$$
\begin{aligned}
& \frac{\overline{\mathrm{d} \zeta_{+}}}{\mathrm{d} t}=\mathrm{i} \zeta_{-}\left(\frac{1}{\zeta_{+}^{2}-\zeta_{-}^{2}}+\frac{1}{1+\zeta_{-}^{2}}\right) \\
& \frac{\overline{d \zeta_{-}}}{d t}=\mathrm{i} \zeta_{+}\left(\frac{1}{\zeta_{-}^{2}-\zeta_{+}^{2}}+\frac{1}{1+\zeta_{+}^{2}}\right) \\
& \frac{\overline{\mathrm{d} \hat{\zeta}_{0}}}{\mathrm{~d} t}=\frac{1}{1+\zeta_{+}^{2}}+\frac{1}{1+\zeta_{-}^{2}}
\end{aligned}
$$

These may be derived from the re-scaled Hamiltonian, cf. (3),

$$
H=-\frac{1}{2} \log \left|\frac{1}{1+\zeta_{+}^{2}}-\frac{1}{1+\zeta_{-}^{2}}\right|
$$

The simplest way to verify that (4a) and (4b) are indeed in canonical form with $H$ as the Hamiltonian is to consider the analytic continuation of $H$, viz.,

$$
\mathcal{H}=-\frac{1}{2} \log \left(\frac{1}{1+\zeta_{+}^{2}}-\frac{1}{1+\zeta_{-}^{2}}\right)
$$

It is then easy to see that Eqs. (4a) and (4b) are simply

$$
\frac{\overline{\mathrm{d} \zeta_{+}}}{\mathrm{d} t}=\mathrm{i} \frac{\partial \mathcal{H}}{\partial \zeta_{-}}, \quad \frac{\overline{\mathrm{d} \zeta_{-}}}{\mathrm{d} t}=\mathrm{i} \frac{\partial \mathcal{H}}{\partial \zeta_{+}} .
$$


Using the analyticity of $\mathcal{H}$, and that $H=\operatorname{Re} \mathcal{H}$, we arrive at the standard Hamiltonian formulation of Eqs. (4a) and (4b).

\section{LEAPFROGGING SOLUTIONS}

We consider leapfrogging motions along the real axis, where the vortices are originally aligned on the $y$-axis. For such motions $\zeta_{0}$ is imaginary and, according to $(2 \mathrm{a}), \zeta_{+}$is real and $\zeta_{-}$imaginary both before and after rescaling. In Eqs. (4a) and (4b) we set

$$
\zeta_{+}(t)=X(t), \quad \zeta_{-}(t)=\mathrm{i} Y(t),
$$

with $X(t)$ and $Y(t)$ real. In terms of these quantities Eqs. (4a) and (4b) become

$$
\begin{aligned}
\frac{\mathrm{d} X}{\mathrm{~d} t} & =-\frac{Y\left(1+X^{2}\right)}{\left(X^{2}+Y^{2}\right)\left(1-Y^{2}\right)}, \\
\frac{\mathrm{d} Y}{\mathrm{~d} t} & =\frac{X\left(1-Y^{2}\right)}{\left(X^{2}+Y^{2}\right)\left(1+X^{2}\right)} .
\end{aligned}
$$

The vortices in both pairs have the real axis as a common axis of symmetry. This discrete symmetry (to flipping about the real axis and reversing the circulations) is preserved throughout the motion.

At the initial instant we have $\zeta_{-}=\frac{1}{2} \Gamma\left(d_{1}-d_{2}\right), \zeta_{0}=\frac{1}{2} \Gamma\left(d_{1}+d_{2}\right)$, where $d_{1,2}$ are both imaginary. In terms of the initial values $X(0)=X_{0}$ and $Y(0)=Y_{0}$

$$
X_{0}=0, \quad Y_{0}=\frac{1-\alpha}{1+\alpha},
$$

where $\alpha=d_{2} / d_{1}$ is the (real) pair separation ratio at $t=0$.

Equations (7b) are nonlinear but integrable by virtue of the existence of an integral of motion, the Hamiltonian, given by (5) specialized to (7a), or

$$
H=-\frac{1}{2} \log \left(\frac{1}{1-Y^{2}}-\frac{1}{1+X^{2}}\right) .
$$

The integral takes the form

$$
\frac{\left(1+X^{2}\right)\left(1-Y^{2}\right)}{X^{2}+Y^{2}}=h=\mathrm{e}^{2 H},
$$

which may also be written

$$
\left(X^{2}+h+1\right)\left(Y^{2}+h-1\right)=h^{2} .
$$

The connection between $\alpha$ and $h$ is given by

$$
\frac{4 \alpha}{(1-\alpha)^{2}}=h \text {. }
$$

It is easy to verify that

$$
\dot{X}=\frac{\partial H}{\partial Y}, \quad \dot{Y}=-\frac{\partial H}{\partial X} .
$$

We note that the equations of motion (7b) can be written

$$
\frac{\mathrm{d} X}{\mathrm{~d} t}=-\frac{h Y}{\left(1-Y^{2}\right)^{2}}, \quad \frac{\mathrm{d} Y}{\mathrm{~d} t}=\frac{h X}{\left(1+X^{2}\right)^{2}} .
$$

Level curves of $H$, Eq. (9a), are plotted in Fig. 3. (An equivalent figure also appears in Love's paper $^{5}$ at the end of Sec. 4.) Close to $(X, Y)=(0,0)$ these curves are circles $X^{2}+Y^{2} \approx h^{-1}$. This limit corresponds to $h \rightarrow \infty$ or $\alpha \rightarrow 1$. We explore it further in Sec. VII.

In order for a curve (9b) to be closed, we must be able to solve (9b) for $X$ when $Y=0$. This implies $h>1$, and all the level curves (9b) for $1<h<\infty$ are closed and lead to leapfrogging 


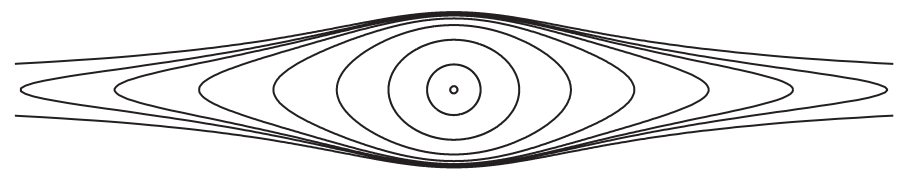

FIG. 3. Level curves of the Hamiltonian (9a).

motions. The lower limit $h=1$ corresponds via (9d) to $\alpha=\alpha_{0}=3-2 \sqrt{2}$. The range in $\alpha$ to be explored, and to which we restrict attention, is then

$$
3-2 \sqrt{2}<\alpha<1 .
$$

The determination of the lower limit of this range, $\alpha_{0}=\sigma^{2}$, is a key result of the classical analyses. ${ }^{4,5}$ See also Appendix B of Ref. 10 for a development of this material in a notation more similar to that used here.

The period $T_{l f}$ of the leapfrogging motion is given by Love ${ }^{5}$ in terms of elliptic integrals. In the present notation, and using the re-scaled time scale occurring in (7b), we have

$$
T_{l f}=2^{6} \frac{\alpha^{2}}{(1-\alpha)^{2}}\left[\frac{E\left(k^{2}\right)}{\left(6 \alpha-\alpha^{2}-1\right)}-\frac{K\left(k^{2}\right)}{(1+\alpha)^{2}}\right],
$$

where $k^{2} \equiv-2^{4} \alpha(1+\alpha)^{2}(1-\alpha)^{-4}$, and $K$ and $E$ are the complete elliptic integrals of the first and second kind, respectively.

\section{LINEAR STABILITY THEORY}

Within the framework of Eqs. (4a) and (4b) we now consider perturbations to the periodic motion described in Sec. III of the form

$$
\begin{aligned}
& \zeta_{+}(t)=X(t)+\epsilon\left[\xi_{+}(t)+\mathrm{i} \eta_{+}(t)\right], \\
& \zeta_{-}(t)=i Y(t)+\epsilon\left[\xi_{-}(t)+\mathrm{i} \eta_{-}(t)\right],
\end{aligned}
$$

where $\epsilon$ is small and determines the size of the perturbation. All the functions $X, Y, \xi_{ \pm}$, and $\eta_{ \pm}$ appearing here are real.

The most transparent way to derive the linearized perturbation equations is to start from Eqs. (6b) and expand to linear order. This gives

$$
\begin{aligned}
& \dot{\xi}_{+}-\mathrm{i} \dot{\eta}_{+}= \\
& \mathrm{i} \frac{\partial^{2} \mathcal{H}}{\partial \zeta_{-}^{2}}\left(\xi_{-}+\mathrm{i} \eta_{-}\right)+\mathrm{i} \frac{\partial^{2} \mathcal{H}}{\partial \zeta_{+} \partial \zeta_{-}}\left(\xi_{+}+\mathrm{i} \eta_{+}\right), \\
& \dot{\xi}_{-}-\mathrm{i} \dot{\eta}_{-}= \\
& \mathrm{i} \frac{\partial^{2} \mathcal{H}}{\partial \zeta_{+}^{2}}\left(\xi_{+}+\mathrm{i} \eta_{+}\right)+\mathrm{i} \frac{\partial^{2} \mathcal{H}}{\partial \zeta_{-} \partial \zeta_{+}}\left(\xi_{-}+\mathrm{i} \eta_{-}\right) .
\end{aligned}
$$

The derivatives of $\mathcal{H}$ for the base solution, $\zeta_{+}=X, \zeta_{-}=\mathrm{i} Y$, are

$$
\begin{aligned}
\frac{\partial^{2} \mathcal{H}}{\partial \zeta_{-}^{2}} & =-\frac{\partial^{2} H}{\partial Y^{2}}=-H_{Y Y}, \\
\frac{\partial^{2} \mathcal{H}}{\partial \zeta_{+}^{2}} & =\frac{\partial^{2} H}{\partial X^{2}}=H_{X X}, \\
\frac{\partial^{2} \mathcal{H}}{\partial \zeta_{-} \partial \zeta_{+}} & =-i \frac{\partial^{2} H}{\partial X \partial Y}=-i H_{X Y} .
\end{aligned}
$$


The components of the Hessian of $H$,

$$
\mathcal{W}=\left[\begin{array}{ll}
H_{X X} & H_{X Y} \\
H_{Y X} & H_{Y Y}
\end{array}\right]
$$

are, of course, all real. Explicitly,

$$
H_{X Y}=H_{Y X}=\frac{2 X Y}{\left(X^{2}+Y^{2}\right)^{2}}
$$

and

$$
H_{X X}=F(\mathrm{i} Y, X), \quad H_{Y Y}=-F(X, \mathrm{i} Y),
$$

where

$$
F\left(Z_{1}, Z_{2}\right)=\frac{\left(1+Z_{1}^{2}\right)\left(3 Z_{2}^{4}-Z_{1}^{2} Z_{2}^{2}+Z_{1}^{2}+Z_{2}^{2}\right)}{\left(1+Z_{2}^{2}\right)^{2}\left(Z_{1}^{2}-Z_{2}^{2}\right)^{2}} .
$$

Separating (13a) into real and imaginary parts, we now see that the perturbations decouple into two independent systems of equations for two variables each,

$$
\begin{aligned}
& \frac{d}{d t}\left[\begin{array}{l}
\xi_{+} \\
\eta_{-}
\end{array}\right]=\left[\begin{array}{rr}
H_{X Y} & H_{Y Y} \\
-H_{X X} & -H_{X Y}
\end{array}\right]\left[\begin{array}{l}
\xi_{+} \\
\eta_{-}
\end{array}\right], \\
& \frac{d}{d t}\left[\begin{array}{l}
\xi_{-} \\
\eta_{+}
\end{array}\right]=\left[\begin{array}{ll}
H_{X Y} & -H_{X X} \\
H_{Y Y} & -H_{X Y}
\end{array}\right]\left[\begin{array}{l}
\xi_{-} \\
\eta_{+}
\end{array}\right] .
\end{aligned}
$$

We note that the coefficient matrix appearing in the second of these is

$$
\left[\begin{array}{ll}
H_{X Y} & -H_{X X} \\
H_{Y Y} & -H_{X Y}
\end{array}\right]=\mathcal{W E}
$$

where

$$
\mathcal{E}=\left[\begin{array}{rr}
0 & -1 \\
1 & 0
\end{array}\right]
$$

We note for later use that $\mathcal{E}^{2}=-\mathbf{1}$.

The coefficient matrix appearing in Eq. (15a) is the transpose,

$$
\left[\begin{array}{rr}
H_{X Y} & H_{Y Y} \\
-H_{X X} & -H_{X Y}
\end{array}\right]=(\mathcal{W E})^{T}=\mathcal{E}^{T} \mathcal{W}=-\mathcal{E} \mathcal{W} .
$$

The perturbation equations may thus be written in the form

$$
\frac{\mathrm{d}}{\mathrm{d} t}\left[\begin{array}{c}
\xi_{+} \\
\eta_{-}
\end{array}\right]=-\mathcal{E} \mathcal{W}\left[\begin{array}{c}
\xi_{+} \\
\eta_{-}
\end{array}\right], \quad \frac{\mathrm{d}}{\mathrm{d} t}\left[\begin{array}{c}
\xi_{-} \\
\eta_{+}
\end{array}\right]=\mathcal{W E}\left[\begin{array}{c}
\xi_{-} \\
\eta_{+}
\end{array}\right],
$$

where $\mathcal{W}$ is the Hessian (13c) and $\mathcal{E}$ is given by (16). The following explicit form is also important

$$
\frac{d}{d t}\left[\begin{array}{c}
\xi_{+} \\
\eta_{-}
\end{array}\right]=\mathcal{A}^{T}\left[\begin{array}{c}
\xi_{+} \\
\eta_{-}
\end{array}\right], \quad \frac{d}{d t}\left[\begin{array}{c}
\xi_{-} \\
\eta_{+}
\end{array}\right]=\mathcal{A}\left[\begin{array}{c}
\xi_{-} \\
\eta_{+}
\end{array}\right],
$$

where

$$
\mathcal{A}=g(X, Y)\left[\begin{array}{cc}
X Y & f(i Y, X) \\
f(X, i Y) & -X Y
\end{array}\right],
$$


with (cf. (14a))

$$
g(X, Y)=\frac{2}{\left(X^{2}+Y^{2}\right)^{2}}
$$

and (cf. (14c))

$$
f\left(Z_{1}, Z_{2}\right)=-\frac{1}{2}\left(Z_{1}^{2}-Z_{2}^{2}\right)^{2} F\left(Z_{1}, Z_{2}\right)
$$

Since $g(X, Y)>0$ it may be absorbed into a rescaling of time via $d \tau=g(X, Y) d t$.

Formally the equations for $\left(\xi_{+}, \eta_{-}\right)$and $\left(\xi_{-}, \eta_{+}\right)$appear to be quite similar. There is, however, an important difference in terms of the physics between the two types of perturbations: The $\left(\xi_{+}, \eta_{-}\right)$perturbations preserve the discrete symmetry of the leapfrogging motion since $\zeta_{+}$remains real and $\zeta_{-}$imaginary. In other words, infinitesimal perturbations of this kind must lead from one leapfrogging motion to another. The $\left(\xi_{-}, \eta_{+}\right)$perturbations break the discrete symmetry. Hence, it is among these perturbations that we are to seek potential instabilities of leapfrogging motion.

By construction all perturbations $\left(\xi_{+}, \eta_{-}\right)$and $\left(\xi_{-}, \eta_{+}\right)$conserve the linear impulse $\zeta_{0}$. The change in the analytic continuation of the Hamiltonian, $\mathcal{H}$ in Eq. (6a), for a general perturbation (12) is

$$
\begin{array}{r}
\delta \mathcal{H}=H_{X}\left(\xi_{+}+\mathrm{i} \eta_{+}\right)-\mathrm{i} H_{Y}\left(\xi_{-}+\mathrm{i} \eta_{-}\right)= \\
H_{X} \xi_{+}+H_{Y} \eta_{-}+\mathrm{i}\left(H_{X} \eta_{+}-H_{Y} \xi_{-}\right) .
\end{array}
$$

This is pure imaginary for perturbations with $\xi_{+}=\eta_{-}=0$ which shows that to leading order there is no change in the real part of $\mathcal{H}$, in other words no change in $H$, Eq. (5), for a $\left(\xi_{-}, \eta_{+}\right)$-perturbation. The $\left(\xi_{+}, \eta_{-}\right)$perturbations will in general change $H$, the one exception being a perturbation along $\left(H_{Y},-H_{X}\right)$, i.e., along $(\dot{X}, \dot{Y})$. As we shall see, such perturbations are, in fact, solutions of the linearized equations (15a).

Turning to the angular impulse, we have

$$
\begin{aligned}
I=\Gamma\left(\left|z_{1}^{+}\right|^{2}+\left|z_{2}^{+}\right|^{2}\right. & \left.-\left|z_{1}^{-}\right|^{2}-\left|z_{2}^{-}\right|^{2}\right) \\
& =2 \operatorname{Re}\left(\hat{\zeta}_{0} \overline{\zeta_{0}}+\zeta_{+} \overline{\zeta_{-}}\right),
\end{aligned}
$$

according to Eq. (3.15) of Ref. 10. After rescaling this becomes $I=2 \operatorname{Im}\left(\hat{\zeta}_{0}\right)+2 \operatorname{Re}\left(\zeta_{+} \overline{\zeta_{-}}\right)$. The change in $I$ to first order in a perturbation of the form (12) is

$$
\begin{array}{r}
\delta I=2 \operatorname{Im}\left(\delta \hat{\zeta}_{0}\right)+2 \operatorname{Re}\left[-\mathrm{i} Y\left(\xi_{+}+\mathrm{i} \eta_{+}\right)+X\left(\xi_{-}-\mathrm{i} \eta_{-}\right)\right] \\
=2 \operatorname{Im}\left(\delta \hat{\zeta}_{0}\right)+2\left(X \xi_{-}+Y \eta_{+}\right) .
\end{array}
$$

Thus, $\left(\xi_{+}, \eta_{-}\right)$-perturbations automatically preserve $I$. For $\left(\xi_{-}, \eta_{+}\right)$-perturbations we may preserve $I$ if we agree to move the origin of coordinates, i.e., shift $\delta \hat{\xi}_{0}$, such that $\delta I=0$ for the perturbed initial state. This is possible since $\hat{\zeta}_{0}$ is simply twice the geometrical centroid of the four points where the vortices are located. Such a shift of the origin of coordinates has no effect on the value of the linear impulse or the Hamiltonian, and the value of $\hat{\zeta}_{0}$ does not enter into the dynamical equations for $\zeta_{ \pm}$. Perturbations $\left(\xi_{-}, \eta_{+}\right)$along $(-Y, X)$ preserve $I$ without need for shifting the origin of coordinates.

In summary, then, the perturbations $\left(\xi_{-}, \eta_{+}\right)$may be considered to take place at fixed values of the integrals of motion $H$ and $I$. The perturbations $\left(\xi_{+}, \eta_{-}\right)$have a one-dimensional subspace that does not conserve $H$.

\section{FLOQUET ANALYSIS}

Exploring the solutions to a system such as (17) leads us directly to Floquet theory. ${ }^{8}$ Due to the periodic time dependence of the coefficient matrix, the behavior of solutions is not immediately given by the local behavior of the solutions but rather by these solutions integrated over a period of the periodic motion. In effect, Floquet theory exploits the properties of the return map of the linear system integrated over a period of the motion whose stability is the object of study. We outline the 
basics of the theory in order to establish our notation and emphasize what we need in the present case. The theory is treated in several places in the literature, e.g., Ref. 9.

We are dealing with a general system of ODEs of the form

$$
\dot{\xi}=\mathcal{A} \xi
$$

where $\xi$ is a two-component vector and $\mathcal{A}$ a $2 \times 2$ matrix that is periodic in time. We shall apply the theory to both the $\left(\xi_{+}, \eta_{-}\right)$- and $\left(\xi_{-}, \eta_{+}\right)$-perturbations of Sec. IV.

Construct a so-called fundamental matrix, $\Xi$, by placing in the first column the solution $\xi=(\xi, \eta)$ to (19) with initial condition $(1,0)$. In the second column place the solution with initial condition $(0,1)$. The $2 \times 2$ matrix, $\Xi$ then satisfies the equation of evolution

$$
\dot{\Xi}=\mathcal{A} \Xi
$$

If $\mathcal{A}$ were constant in time, the solution would be easy enough,

$$
\Xi(t)=\mathrm{e}^{\mathcal{A} t} \Xi(0) \text {. }
$$

If $\mathcal{A}$ has eigenvalues $\mu_{1,2}$ with corresponding eigenvectors $\boldsymbol{v}^{(1,2)}$, we have

$$
\mathcal{A} \boldsymbol{v}^{(1,2)}=\mu_{1,2} \boldsymbol{v}^{(1,2)}, \quad \mathrm{e}^{\mathcal{A} t} \boldsymbol{v}^{(1,2)}=\mathrm{e}^{\mu_{1,2} t} \boldsymbol{v}^{(1,2)} .
$$

Hence, expanding $\boldsymbol{\xi}(0)$ along $\boldsymbol{v}^{(1,2)}$, we obtain solution components that vary as $\mathrm{e}^{\mu_{1,2} t}$. If $\mu_{1,2}$ is pure imaginary, this leads to oscillatory evolution and thus spectral stability. On the other hand, if either of $\mu_{1,2}$ has a positive real part, we obtain unstable exponential growth of the perturbation.

For a time-periodic coefficient matrix we must proceed a bit differently: We exploit the periodicity of $\mathcal{A}$ with some period, $T$, to argue that if $\Xi(t)$ is a solution of (20), then $\Xi(t+T)$ will also be a solution. Next, since the space of solution vectors is two-dimensional, the columns of $\Xi(t+T)$ may be expressed as linear combinations of the columns of $\Xi(t)$. In other words, there exists a matrix, $M$, called the monodromy matrix, such that

$$
\Xi(t+T)=\Xi(t) M .
$$

With the two independent solutions we have chosen, we have $\Xi(0)=1$, the $2 \times 2$ unit matrix. Hence,

$$
M=\Xi(T), \quad \Xi(t+T)=\Xi(t) \Xi(T) .
$$

If we wish to find the solution $\hat{\Xi}(t)$ starting from general initial conditions $\hat{\Xi}(0)=\mathcal{C}$, we have only to set $\hat{\Xi}(t)=\Xi(t) \mathcal{C}$. Then,

$$
\dot{\hat{\Xi}}=\dot{\Xi} \mathcal{C}=\mathcal{A} \Xi \mathcal{C}=\mathcal{A} \hat{\Xi},
$$

so $\hat{\Xi}$ satisfies the ODE, and by construction $\hat{\Xi}(0)=\mathcal{C}$.

A word of caution is necessary if $\Xi(t)$ becomes degenerate. Indeed, the term "fundamental matrix" is usually reserved for the case when $\Xi(t)$ is non-degenerate. If the columns of $\Xi(t)$ become proportional, we must supplement the first column vector by a perpendicular vector to have a basis in the $2 \mathrm{D}$ space. The general theory is then somewhat modified. We continue with the assumption that $\Xi(t)$ is non-degenerate for $0 \leq t \leq T$ and return to the degenerate case as necessary.

Let $\boldsymbol{v}^{( \pm)}$be the eigenvectors of $M$ corresponding to eigenvalues $\rho_{ \pm}$, respectively, i.e., $M \boldsymbol{v}^{( \pm)}$ $=\rho_{ \pm} \boldsymbol{v}^{( \pm)}$. It is important to emphasize that $\rho_{ \pm}$and $\boldsymbol{v}^{( \pm)}$are time independent quantities. Important properties of $\rho_{ \pm}$follow from the identity

$$
\frac{\mathrm{d}}{\mathrm{d} t} \operatorname{det} \Xi=\operatorname{Tr} \mathcal{A} \operatorname{det} \Xi
$$

This is, essentially, the relation for change of area in the 2D "flow" (20) and is, in any event, not difficult to verify. In our case the coefficient matrix $\mathcal{A}$ is either $\mathcal{W E}$ or its transpose. Both have vanishing trace, so the right-hand side of (22) vanishes, and det $\Xi$ is invariant in time. Since det $\Xi(0)$ $=1$, we have $\operatorname{det} M=\operatorname{det} \Xi(T)=1$ and thus $\rho_{+} \rho_{-}=1$. Furthermore, in our case the vectors and matrices are all real. In particular, the matrix $M$ is real and its eigenvalues are thus either both real 
(in which case they have the form $\rho_{ \pm}=\rho^{ \pm 1}$ for some real $\rho$ ), or they are complex conjugates (in which case they have the form $\rho_{ \pm}=\mathrm{e}^{ \pm i \varphi}$ for some angle $\varphi$ ).

Now consider the time-dependent vectors

$$
\boldsymbol{\xi}^{( \pm)}(t)=\Xi(t) \boldsymbol{v}^{( \pm)}
$$

We have

$$
\dot{\boldsymbol{\xi}}^{( \pm)}=\dot{\Xi} \boldsymbol{v}^{( \pm)}=\mathcal{A} \Xi \boldsymbol{v}^{( \pm)}=\mathcal{A} \xi^{( \pm)},
$$

so the $\xi^{( \pm)}(t)$ are solutions to the ODEs (19). They have the initial values $\xi^{( \pm)}(0)=\boldsymbol{v}^{( \pm)}$. We see that

$$
\begin{aligned}
& \xi^{( \pm)}(t+T)=\Xi(t+T) \boldsymbol{v}^{( \pm)}= \\
& \Xi(t) M \boldsymbol{v}^{( \pm)}=\rho_{ \pm} \Xi(t) \boldsymbol{v}^{( \pm)}=\rho_{ \pm} \xi^{( \pm)}(t) .
\end{aligned}
$$

Thus, over a period of the coefficient matrix $\mathcal{A}$ the solutions $\xi^{( \pm)}$are multiplied by $\rho_{ \pm}$, respectively. The general solution to the functional relation (24) is

$$
\xi^{( \pm)}(\tau)=\rho_{ \pm}^{\tau / T} \Pi_{ \pm}(\tau)
$$

where the arbitrary function $\boldsymbol{\Pi}_{ \pm}(\tau)$ is periodic with period $T$ and satisfies $\boldsymbol{\Pi}_{ \pm}(0)=\boldsymbol{v}^{( \pm)}$. A direct proof that $\Pi_{ \pm}(\tau)$ is periodic follows:

$$
\begin{aligned}
& \Pi_{ \pm}(\tau+T)=\rho_{ \pm}^{-(\tau+T) / T} \boldsymbol{\xi}^{( \pm)}(\tau+T)= \\
& \quad \rho_{ \pm}^{-(\tau+T) / T} \rho_{ \pm} \boldsymbol{\xi}^{( \pm)}(\tau)=\rho_{ \pm}^{-\tau / T} \boldsymbol{\xi}^{( \pm)}(\tau)=\Pi_{ \pm}(\tau) .
\end{aligned}
$$

The quantities $\rho_{ \pm}$are called the Floquet multipliers. One often sets $\rho_{ \pm}=\mathrm{e}^{\mu_{ \pm} T}$, even though the $\mu_{ \pm}$are defined only up to multiples of $2 \pi i / T$. The $\mu_{ \pm}$are called the Floquet exponents.

If $\rho_{ \pm}$are reciprocal real numbers, we have found a solution to (19) that grows exponentially. The underlying periodic motion from which these equations arose as linear perturbations is then unstable. If $\rho_{ \pm}$are complex conjugates of modulus 1 , the motion is linearly stable. A general initial condition may be decomposed along $\boldsymbol{v}^{( \pm)}$,

$$
\boldsymbol{\xi}(0)=a_{+} \boldsymbol{v}^{(+)}+a_{-} \boldsymbol{v}^{(-)}
$$

and so will evolve according to

$$
\begin{aligned}
\boldsymbol{\xi}(\tau)= & a_{+} \boldsymbol{\xi}^{(+)}(\tau)+a_{-} \boldsymbol{\xi}^{(-)}(\tau)= \\
& a_{+} \rho_{+}^{\tau / T} \boldsymbol{\Pi}^{(+)}(\tau)+a_{-} \rho_{-}^{\tau / T} \boldsymbol{\Pi}^{(-)}(\tau) .
\end{aligned}
$$

\section{FLOQUET ANALYSIS APPLIED TO THE EQUATIONS OF SEC. IV}

We now adapt the general theory to our perturbation equations for the leapfrogging motion. First, concerning the period, $T$, of the coefficient matrices, $-\mathcal{E} \mathcal{W}$ and $\mathcal{W E}$ in Eqs. (17), we see that this must be half the period of the leapfrogging motion $T_{l f}$, (11). We start at $X=0$ and some finite value of $Y$. During an entire cycle of the leapfrogging motion $X$ and $Y$ vary through positive and negative values. However, all the quantities $H_{X X}, H_{X Y}, H_{Y Y}$ appearing in the coefficient matrices of the stability problem are even functions of $X$ and $Y$. Hence, the period of the matrices is half the leapfrogging period.

Let us first consider the $\left(\xi_{+}, \eta_{-}\right)$equations even though these are not of primary interest to the stability of leapfrogging. There is one obvious solution to these equations: If we differentiate Eqs. (9e) once more with respect to time, we get

$$
\ddot{X}=H_{Y X} \dot{X}+H_{Y Y} \dot{Y}, \quad \ddot{Y}=-H_{X X} \dot{X}-H_{X Y} \dot{Y} .
$$

Written in matrix form, these equations show that $\left(\xi_{+}, \eta_{-}\right)=(\dot{X}, \dot{Y})$ solve Eq. (15a). 
The initial conditions (8) for leapfrogging imply

$$
\dot{X}(0)=\frac{-1}{h Y_{0}^{3}}, \quad \dot{Y}(0)=0 .
$$

This follows from $(7 b)$ or $\left(7 b^{\prime}\right)$ and $(9 b)$ when the values from (8) are substituted. It is also clear from Fig. 3 that $\dot{Y}(0)$ vanishes and that $\dot{X}(0)$ is negative: The leapfrogging motion starts at a point on the positive $Y$-axis of Fig. 3 as given by Eqs. (8). Since the level curves all have horizontal tangents on this axis, a $\left(\xi_{+}, \eta_{-}\right)$-perturbation of the initial condition that has only an $X$-component will simply move the phase point a bit forward or backward along the chosen trajectory, i.e., lead to a leapfrogging motion with the same value of the Hamiltonian. At time $T$ the perturbation $(\dot{X}, \dot{Y})$ will have evolved into $(\dot{X}(T), \dot{Y}(T))=(-\dot{X}(0), 0)$. In other words, the $\left(\xi_{+}, \eta_{-}\right)$-perturbation that at $t=0$ is $(1,0)$ must, by linearity, have evolved into $(-1,0)$ at time $t=T$. This gives the first column of the monodromy matrix $M$.

Next, from the determinant of the monodromy matrix being +1 we know that the lower diagonal element must also be -1 . Hence, we have the form of the monodromy matrix for the $\left(\xi_{+}\right.$, $\eta_{-}$) equations,

$$
M=\left[\begin{array}{cc}
-1 & A \\
0 & -1
\end{array}\right] \text {. }
$$

To find the number $A$, we proceed as follows. We consider a perturbation with initial condition $\left(\xi_{+}\right.$, $\left.\eta_{-}\right)=(0,1)$ of a state with initial condition $(X, Y)=\left(0, Y_{0}\right)$. This effectively takes us to another leapfrogging motion with initial condition $(X, Y)=\left(0, Y_{0}+\epsilon\right)$. Now, the leapfrogging period has increased by an amount $\mathrm{d} T_{l f}=\epsilon \partial T_{l f} / \partial Y_{0}$, which can be computed from (8) and (11). So after a time $T$ $=T_{l f} / 2$, the perturbed system will be a time $\mathrm{d} T_{l f} / 2$ behind reaching its own half-period. To first order in $\epsilon$, the system will have evolved to $(X, Y)=\left(-\mathrm{d} T_{l f} \dot{X}(T) / 2,-Y_{0}-\epsilon\right)$. Since the perturbation governed by (15a) must evolve in agreement with this result to linear order in $\epsilon$, we will have $\left(\xi_{+}, \eta_{-}\right)=\left(-\mathrm{d} T_{l f} \dot{X}(T) / 2,-1\right)$ at time $T$. Now, $\dot{X}(T)=-\dot{X}(0)$, so

$$
A=\frac{1}{2} \frac{\partial T_{l f}}{\partial Y_{0}} \dot{X}(0)
$$

with $A<0$. The result (27) and (28) shows that leapfrogging is always stable to $\left(\xi_{+}, \eta_{-}\right)$perturbations, as we have already argued. Also, it has been useful in checking our numerical procedure for evaluating the monodromy matrix.

It is straightforward to compute the Floquet exponents for the leapfrogging motion numerically. We have done so as a function of the parameter $\alpha$ in the initial condition (8). The classical leapfrogging solution is required. This solution is given in terms of elliptic functions ${ }^{4,5}$ that would, in any event, require numerical evaluation. It is therefore easier operationally, and just as accurate, to simply solve the ODEs (7b) numerically along with the stability equations (17) such that $X(t)$ and $Y(t)$ are available at each step. We have used the Runge-Kutta 45 solver in the software package MATLAB ${ }^{\circledR}$ for this purpose.

For the $\left(\xi_{+}, \eta_{-}\right)$-equations the numerical calculations simply verify the result in (27) and (28). The Floquet analysis calculation for the $\left(\xi_{-}, \eta_{+}\right)$-equations is more interesting. The results have been collected in Fig. 4 which shows both $\mu_{+} T$ and $\mu_{+}$as functions of $\alpha$. The relevant interval is $\alpha_{0}<\alpha<1$. In this interval $\alpha_{0}=\sigma^{2} \approx 0.172$ marks the onset of leapfrogging. Since $T \rightarrow \infty$ as $\alpha \rightarrow \alpha_{0}$, we have plotted both $\mu_{+}$and $\mu_{+} T$ in Fig. 4 as functions of $\alpha$. We see that for a range of $\alpha$, from the onset of leapfrogging to $\alpha=\alpha_{2} \approx 0.382$, we have a real, positive Floquet exponent corresponding to instability. The Floquet exponent $\mu_{+}$vanishes at $\alpha=\alpha_{0}$ and at $\alpha=\alpha_{2}$. Beyond $\alpha=\alpha_{2}$ the Floquet exponents become pure imaginary. The positive imaginary part, $\operatorname{Im} \mu_{+} T$, has been plotted in Fig. 4. Again, since $T \rightarrow 0$ for $\alpha \rightarrow 1$, we have also plotted $\operatorname{Im} \mu_{+}$. Figure 5 shows a magnification of the region close to $\alpha=\alpha_{2}$. The vertical line is at $\alpha_{2}=0.38197 \ldots$, which we determine analytically in the sequel. This value agrees to three decimal places with the value found 


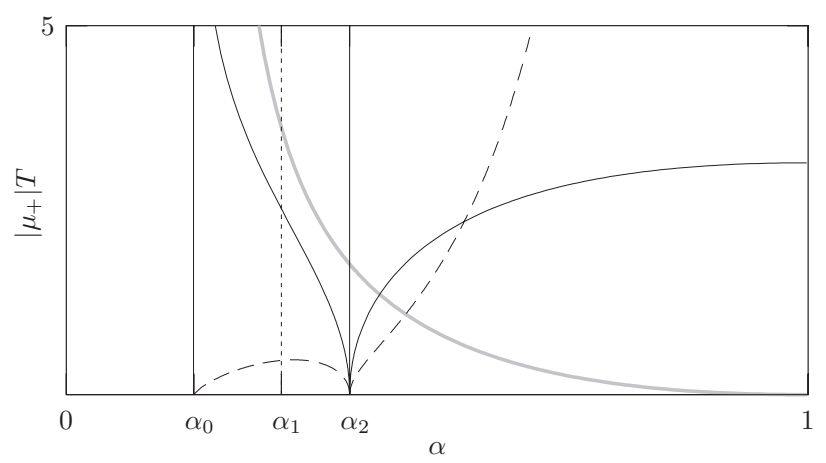

FIG. 4. Floquet exponents as a function of $\alpha$ shown for $\alpha_{0}<\alpha \leq \alpha_{2}$. See the text for these values of $\alpha$. The dashed curve shows $2\left|\mu_{+}\right|$, the thick grey curve shows $T / 3$, cf. (11), and the solid curve shows $\left|\mu_{+}\right| T$. Note that $\mu_{+}$is purely real for $\alpha<\alpha_{2}$ and purely imaginary for $\alpha_{2}<\alpha<1$, indicating that the monodromy matrix has complex conjugate eigenvalues of unit modulus in the latter range.

by Acheson ${ }^{6}$ using direct numerical experiments. It also agrees to all decimal places with the value of $\phi^{2}$, where $\phi$ is the golden ratio. The numerical result motivates the analytical argument that the zero Floquet exponent, in fact, occurs for $\alpha=\phi^{2}$ given below.

The Floquet exponent plot in Fig. 4 shows an inflection point, at $\alpha=\alpha_{1} \approx 0.29$, approximately the point where Acheson 6 noticed a transition from the "walkabout" to the disintegration mode of instability by numerical experiments. However, it is optimistic to expect a linearized analysis to detect a crossover between two modes of finite-amplitude instability. Furthermore, our numerical investigation shows that the transition from "walkabout" to disintegration mode is not sharp in the same sense as the transition at $\alpha=\alpha_{2}$. Figure 6 shows an orbit of two vortex pairs scattering off each other through an intermediate stage of leapfrogging motion. This is similar to scattering processes discussed in Ref. 7. The orbit realised in Fig. 6 was realised by integrating both forward and backward in time from a perturbed leapfrogging state with $\alpha=0.25$. The two directions are equivalent, in the sense that moving backwards in time from a state perturbed by $\left(\xi_{-}, \eta_{+}\right)$corresponds to moving forward from a different perturbation $\left(\xi_{-},-\eta_{+}\right)$. This shows that one single leapfrogging state can be pushed into either the "walkabout" or the "disintegration" instability mode by different small perturbations. We conclude that the transition is a gradual one, where one tends to see disintegration instability for $\alpha_{1}<\alpha<0.26$ and "walkabout" instability for $0.26<\alpha<\alpha_{2}$.

We proceed to give a tentative argument as to the exact value of the $\alpha_{2}$ marking the transition to instability. Consider a set of trajectories of the form, cf. (25),

$$
\xi(\tau, \alpha)=e^{\mu(\alpha) \tau} \Pi(\tau, \alpha)
$$

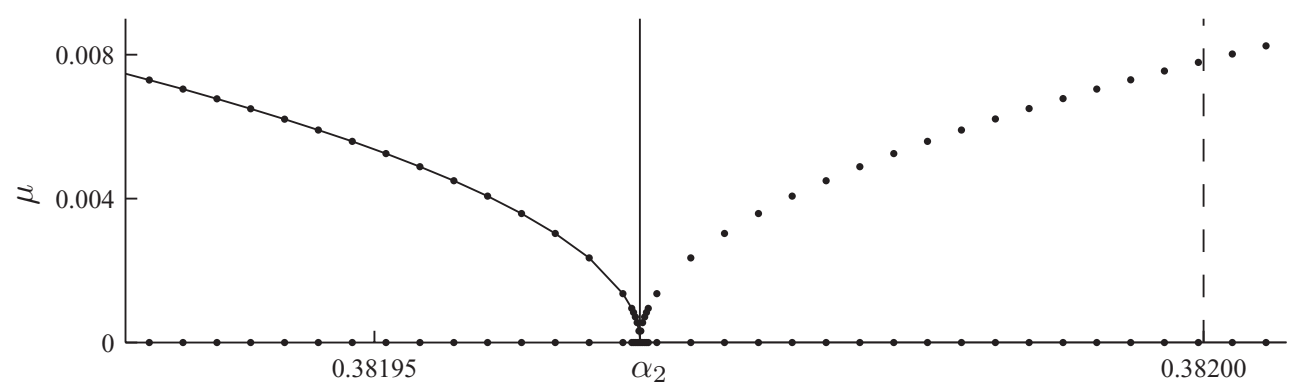

FIG. 5. Detail of the Floquet exponent $\mu_{+}$around $\alpha=\alpha_{2}=\phi^{2}$. For $\alpha<\alpha_{2}$, where $\mu_{+}$is imaginary, we show $\mu_{+} / i$. The dashed line at $\alpha=0.382$ shows Acheson's numerically determined value for the crossover from unstable to stable leapfrogging. ${ }^{6}$ The text provides further discussion. 


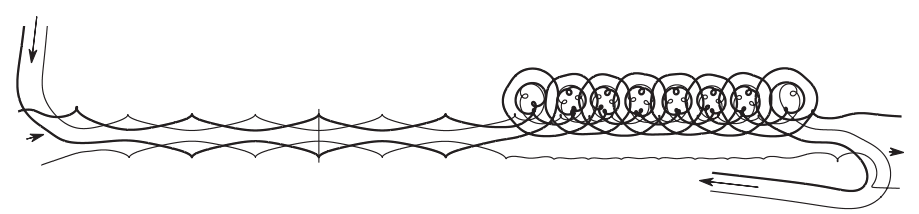

FIG. 6. Scattering of two identical vortex pairs showing intermediate states consisting of leapfrogging and "walkabout" motions. The vertical bar marks the starting leapfrogging configuration, $\alpha=0.25,\left(\xi_{-}, \eta_{+}\right)=(-1,1) \times 10^{-5}$, from which time is integrated forwards and backwards. The computation has been checked by reverse integration, and the variation of the integrals of motion is of negligible order $\left(10^{-12}\right)$.

as a one-parameter family of curves in $\mathbb{R}^{2}$ by the parameter $\alpha . \mu$ is one of the Floquet exponents, and $\boldsymbol{\Pi}$ is periodic. We boldly assume the function $\boldsymbol{\xi}_{\alpha}$, where subscript denotes differentiation, exists, in which case

$$
\xi_{\alpha}=\tau \mu_{\alpha} \xi+e^{\mu \tau} \Pi
$$

Now, it is clear that $\mu(\alpha)$ undergoes a sharp transition at $\alpha=\alpha_{2}$, with distinct limiting values of the derivative $\mu_{\alpha}$ on either side of the transition point. From Fig. 4, we see that $\mu_{\alpha}$ diverges, so we expect $\boldsymbol{\xi}_{\alpha},(29)$, to change dramatically at the transition point. If we differentiate (19) with respect to $\alpha$, we obtain

$$
\dot{\xi}_{\alpha}=\mathcal{A}_{\alpha} \xi+\mathcal{A} \xi_{\alpha} .
$$

This dynamical system is identical to the one governing the evolution of $\boldsymbol{\xi}$, except for the forcing term $\mathcal{A}_{\alpha} \xi$.

We are thus naturally led to examine the behavior of the matrix $\mathcal{A}_{\alpha}$. In particular the determinant $\left|\mathcal{A}_{\alpha}\right|$ will be of interest. We note that $\left|\mathcal{A}_{\alpha}\right|$ is a smooth function of time and periodic with period $T$. It has two distinct regimes for $\alpha_{0}<\alpha<1$, with the transition taking place for a particular value of $\alpha$, say $\alpha=\alpha_{2}^{\prime}$. For $\alpha<\alpha_{2}^{\prime},\left|\mathcal{A}_{\alpha}\right|$ changes sign twice during one period. For $\alpha_{2}^{\prime}<\alpha,\left|\mathcal{A}_{\alpha}\right|$ is positive throughout the period. So $\alpha_{2}^{\prime}$ marks a distinct change in the character of the forcing term in (30). The crossover takes place at the time corresponding to $X=0$, so we can determine $\alpha_{2}^{\prime}$ by differentiating the matrix,

$$
\left[\begin{array}{cc}
X Y & f(i Y, X) \\
f(X, i Y) & -X Y
\end{array}\right],
$$

cf. (18b), with respect to $Y$ at $X=0$ (which is equivalent to differentiating with respect to $Y_{0}$, and so with respect to $\alpha$ ), we find after a brief calculation

$$
\begin{gathered}
\frac{\partial}{\partial Y}\left[\begin{array}{cc}
X Y & f(i Y, X) \\
f(X, i Y) & -X Y
\end{array}\right]_{X=0}= \\
{\left[\begin{array}{cc}
0 & \frac{Y_{0}\left(1-5 Y^{2}\right)}{\left(1-Y_{0}^{2}\right)^{3}} \\
Y_{0}\left(1-2 Y_{0}^{2}\right) & 0
\end{array}\right] .}
\end{gathered}
$$

Thus, in order for $\mathcal{A}_{\alpha}$ to have a null vector we find the necessary condition

$$
\left(1-2 Y_{0}^{2}\right)\left(1-5 Y_{0}^{2}\right)=0 .
$$

In other words,

$$
Y_{0}=\frac{1}{\sqrt{2}} \quad \text { or } \quad Y_{0}=\frac{1}{\sqrt{5}} .
$$

These translate into the values $\alpha=\alpha_{0}=\sigma^{2}$ and $\alpha=\alpha_{2}^{\prime}=\phi^{2}$, respectively. 
The latter corresponds to a high precision to the transition point located by numerical methods, cf. Figs. 4 and 5. It seems the change in the forcing term, i.e., in $\left|\mathcal{A}_{\alpha}\right|$ is somehow critical in determining the transition to instability, and the range $\alpha_{2}^{\prime}<\alpha$ with $\left|\mathcal{A}_{\alpha}\right| \supsetneqq 0$ corresponds to linearly stable leapfrogging. We thus speculate that $\alpha_{2}=\alpha_{2}^{\prime}$.

\section{THE $\alpha \rightarrow 1$ LIMIT}

When $\alpha \rightarrow 1$ the two positive and the two negative vortices are close and orbit one another in bound states, effectively producing a vortex pair with strengths $\pm 2 \Gamma$. In this limit $X^{2}$ and $Y^{2}$ are $\ll 1$. Thus Eqs. (7b) reduce to

$$
\frac{\mathrm{d} X}{\mathrm{~d} t}=-\frac{Y}{X^{2}+Y^{2}}, \quad \frac{\mathrm{d} Y}{\mathrm{~d} t}=\frac{X}{X^{2}+Y^{2}} .
$$

One consequence of Eqs. (32) is that $X^{2}+Y^{2}$ is a constant. From its initial value (8), and to leading order in $1-\alpha$,

$$
X^{2}+Y^{2}=\frac{1}{4}(1-\alpha)^{2}=\frac{1}{h}
$$

From the definition of $g(X, Y)$, Eq. (18c), we then have $d \tau=2 h^{2} d t$. In this limit $t$ and re-scaled time, $\tau$, are proportional. The solution to Eqs. (32) that satisfies the initial conditions (8) is

$$
X(t)=-\frac{\sin (h t)}{\sqrt{h}}, \quad Y(t)=\frac{\cos (h t)}{\sqrt{h}} .
$$

In the system matrix (18b) we now get

$$
X Y=-\frac{\sin (2 h t)}{2 h} .
$$

Also, to lowest order in $1-\alpha$, we find

$$
f(X, i Y)=f(X, i Y) \approx-\frac{1}{2}\left(X^{2}-Y^{2}\right)=\frac{\cos (2 h t)}{2 h} .
$$

Thus, Eq. (15b) read

$$
\frac{\mathrm{d}}{\mathrm{d} t}\left[\begin{array}{l}
\xi_{-} \\
\eta_{+}
\end{array}\right]=\omega\left[\begin{array}{rr}
-\sin (2 \omega t) & \cos (2 \omega t) \\
\cos (2 \omega t) & \sin (2 \omega t)
\end{array}\right]\left[\begin{array}{c}
\xi_{-} \\
\eta_{+}
\end{array}\right]
$$

This matrix has periodicity $T=\pi / \omega$ in $t$. Unfortunately, the $\alpha \rightarrow 1$ limit corresponds to a special case of the general theory in Sec. V where the monodromy matrix is degenerate. The single eigenvalue has only a one-dimensional eigenspace.

One can obtain the general solution of (35) in several ways. For example, one may note that the two vectors

$$
\boldsymbol{\xi}^{(1)}(t)=\left[\begin{array}{c}
\cos (\omega t) \\
\sin (\omega t)
\end{array}\right], \quad \boldsymbol{\xi}^{(2)}(t)=\left[\begin{array}{c}
-\sin (\omega t) \\
\cos (\omega t)
\end{array}\right],
$$

satisfy

$$
\frac{d \boldsymbol{\xi}^{(1)}}{d t}=\omega \boldsymbol{\xi}^{(2)}, \quad \frac{d \boldsymbol{\xi}^{(2)}}{d t}=-\omega \boldsymbol{\xi}^{(1)}
$$


Further, if the matrix on the right-hand side of (35), without the factor $\omega$, is designated $\hat{A}$, then

$$
\hat{A} \xi^{(1)}=\xi^{(2)}, \quad \hat{A} \xi^{(2)}=\xi^{(1)} .
$$

If we posit that a vector, $\boldsymbol{\xi}(t)$, solves (35), and if we expand it in terms of $\boldsymbol{\xi}^{(1,2)}$ as

$$
\boldsymbol{\xi}(t)=a(t) \boldsymbol{\xi}^{(1)}(t)+b(t) \boldsymbol{\xi}^{(2)}(t)
$$

with time-dependent coefficient $a(t)$ and $b(t)$, we find the conditions

$$
\frac{\mathrm{d} a}{\mathrm{~d} t}=-2 \omega b, \quad \frac{\mathrm{d} b}{\mathrm{~d} t}=0 .
$$

This shows that such a decomposition requires $b$ to be a constant, $b=C_{1}$, and $a(t)=-2 \omega C_{1} t+C_{2}$, where $C_{2}$ is a second constant. In particular, $a$ and $b$ cannot both be constants, but there must be a secular term in the expansion.

The general solution is

$$
\left[\begin{array}{l}
\xi_{-} \\
\eta_{+}
\end{array}\right]=\left(C_{2}-2 C_{1} \omega t\right)\left[\begin{array}{c}
\cos \omega t \\
\sin \omega t
\end{array}\right]+C_{1}\left[\begin{array}{r}
-\sin \omega t \\
\cos \omega t
\end{array}\right],
$$

with constants $C_{1}$ and $C_{2}$ chosen to match initial conditions. The initial conditions $\xi_{-}=1, \eta_{+}=0$ correspond to $C_{1}=0, C_{2}=1$, the initial conditions $\xi_{-}=0, \eta_{+}=1$ to $C_{1}=1, C_{2}=0$. At $t=T$ $=\pi / \omega$ these have evolved to $(-1,0)$ and $(2 \pi,-1)$, respectively. Thus,

$$
M=\Xi(T)=\left[\begin{array}{cc}
-1 & 2 \pi \\
0 & -1
\end{array}\right] .
$$

The eigenvalue $\rho=-1$ has a one-dimensional eigenspace. The secular term in (37a) reflects this degeneracy of the monodromy matrix.

\section{CONCLUSIONS}

The stability of leapfrogging motion has been investigated using Floquet theory. By studying the Floquet exponents of the linearized perturbation equations, we have confirmed the numerical results of Acheson. ${ }^{6}$ Furthermore, we have argued analytically that the transition to instability occurs when the pair size ratio is exactly $\alpha=\phi^{2}$, the square of the golden ratio, in agreement with numerical results. The linear analysis does not explain the transition between the "walkabout" and "disintegration" instability modes identified by Acheson, ${ }^{6}$ but our numerical calculations has revealed that the transition is gradual rather than sharp, with both modes accessible by perturbation of a single leapfrogging motion. An example was given of leapfrogging occurring as an intermediate state in the chaotic scattering of vortex pairs. ${ }^{7}$ We mention that the advection of particles by the periodic flow due to leapfrogging is chaotic. This has been explored by Péntek et al. ${ }^{11}$

\section{ACKNOWLEDGMENTS}

The presented work was supported in part by a Niels Bohr Visiting Professorship at the Technical University of Denmark sponsored by the Danish National Research Foundation. This paper is dedicated to the memory of Hassan Aref, who passed away during its preparation. He was a friend and a great inspiration.

${ }^{1}$ H. von Helmholtz, "Über Integrale der hydrodynamischen Gleichungen, welche den Wirbelbewegungen entsprechen," J. Reine Angew. Math. 55, 25-55 (1858); P. G. Tait, "On integrals of the hydrodynamical equations, which express vortex-motion," Philos. Mag. 33(4), 485-512 (1867) (in English).

${ }^{2}$ H. Yamada and T. Matsui, "Preliminary study of mutual slip-through of a pair of vortices," Phys. Fluids 21, 292-294 (1978).

${ }^{3}$ B. N. Shashikanth and J. E. Marsden, "Leapfrogging vortex rings: Hamiltonian structure, geometric phases and discrete reduction," Fluid Dyn. Res. 33, 333-356 (2003). 
${ }^{4}$ W. Gröbli, “Spezielle probleme über die Bewegung geradliniger paralleler Wirbelfäden,” Vierteljahrsschr. Naturforsch. Ges. Zür. 22, 37-81, 129-165 (1877).

${ }^{5}$ A. E. H. Love, "On the motion of paired vortices with a common axis," Proc. London Math. Soc. 25, 185-194 (1893).

${ }^{6}$ D. J. Acheson, "Instability of vortex leapfrogging,” Eur. J. Phys. 21, 269-273 (2000).

${ }^{7}$ L. Tophøj and H. Aref, "Chaotic scattering of two identical point vortex pairs revisited," Phys. Fluids 20, 093605 (2008).

${ }^{8}$ G. Floquet, "Sur les équations différentielles linéaires à coefficients périodiques," Ann. Sci. Ec. Normale Super. (2nd series) 12, 47-88 (1883).

${ }^{9}$ A. H. Nayfeh and B. Balachandran, Applied Nonlinear Dynamics-Analytical, Computational and Experimental Methods (Wiley, New York, 1995).

${ }^{10}$ B. Eckhardt and H. Aref, "Integrable and chaotic motions of four vortices II. Collision dynamics of vortex pairs," Philos. Trans. R. Soc. London, Ser. A 326, 655-696 (1988).

${ }^{11}$ A. Péntek, T. Tél, and T. Toroczkai, "Chaotic advection in the velocity field of leapfrogging vortex pairs," J. Phys. A 28, 2191-2216 (1995). 